\title{
Cellulose nanofibre-poly(lactic acid) microcellular foams exhibiting high tensile toughness
}

$\operatorname{AUTHOR}(\mathrm{S})$ :

Dlouhá, Jana; Suryanegara, Lisman; Yano, Hiroyuki

\section{CITATION:}

Dlouhá, Jana ... [et al]. Cellulose nanofibre-poly(lactic acid) microcellular foams exhibiting high tensile to ughness. Reactive and Functional Polymers 2014, 85: 201-207

ISSUE DATE:

2014-12

URL:

http://hdl.handle.net/2433/192899

\section{RIGHT:}

(C) 2014 Elsevier Ltd.; This is not the published version. Please cite only the published version.; この論文は出版社版でありません。引用の際に は出版社版をご確認ご利用ください。 
Cellulose nanofibre-poly(lactic acid) microcellular foams exhibiting high tensile toughness Jana Dlouhá $^{a, b, c *}$, Lisman Suryanegara ${ }^{a, d}$ and Hiroyuki Yano ${ }^{a}$

${ }^{a}$ Research Institute for Sustainable Humanosphere, Kyoto University, 611-0011 Uji-Kyoto, Japan.

${ }^{\mathrm{b}}$ AgroParisTech, UMR 1092 LERFOB, 54000 Nancy, France.

${ }^{\mathrm{c}}$ INRA, UMR 1092 LERFOB, 54280 Champenoux, France.

${ }^{\mathrm{d}}$ Laboratory of Biocomposites, Research and Development Unit for Biomaterials, Indonesian

Institute of Sciences, Jl. Raya Bogor KM.46, Cibinong, Bogor, 16911, Indonesia.

Corresponding Author

*E-mail: jana.dlouha@nancy.inra.fr. Tel: +33-383-397-314. Fax: + 33-383-394-022

ABSTRACT: We report here the morphology and tensile properties of polylactic acid-cellulose nanofibre (PLA-CNF) microcellular nanocomposites. Two types of CNF were used for the nanocomposite preparation, native and surface acetylated CNF (ac-CNF). Samples were foamed in a mould to enable tensile testing. The effect of the mould use on the foam morphology was first assessed by comparison with free foamed samples. We found that the mould affected the cell growth stage of the foaming process in neat PLA foam while its effect was less important in nanocomposites. Stiffening and strengthening effect of CNF was greatly enhanced by foaming when compared to their solid counterparts. The most notable change in tensile properties was however the large increase in strain at break resulting in the high tensile toughness of microcellular PLA-CNF nancomposites. Strain at break increased up to 7.5 times in neat PLA and up to 31.5 times in the foam containing 3\% of CNF. Surface acetylation of CNF significantly affected the properties of foams with $9 \%$ of CNF loading: while foams with ac-CNF were stiffer, foams with native CNF exhibited higher strain at break and so higher overall toughness.

Keywords: Polylactic acid - Cellulose nanofibres - Microcellular nanocomposites Mechanical properties 


\section{Introduction}

Due to its good stiffness and strength, PLA is one of the most promising bio-based polymers however its inherent brittleness and low impact resistance represent an important limitation to its usage and make its processing and transport more expensive compared to petroleum-based polymers [1]. Microcellular foaming may improve the toughness of polymer matrix [2-4] however the overall mechanical properties of foams in general suffer from the density reduction [5]. Synergistic effect of the nanoparticles reinforcement and the toughening by microcells has therefore the potential to produce not only light-weight but also tough and flexible materials [6, 7].

Positive effect of CNF or microfibrillated cellulose (MFC) on the solid PLA tensile modulus and strength is well documented in the literature [8-11]. On the other hand the increase in the overall toughness and strain at break with the MFC addition is in general inexistent or very limited [8-10]. In some particular cases however, an important increase in strain at break after addition of a small content of acetylated MFC [12], TEMPO-oxidized cellulose [13] or organically modified clay nanoparticles [14] was observed. The increase in the PLA strain at break from $8.4 \%$ to $258 \%$ after addition of $1 \%$ of acetylated CNF was explained by the plastic deformation of pre-existing voids (films were prepared by solvent casting) acting in a similar way to cavitated rubber particles in rubber-toughened polymer systems $[15,16]$. Similarly, Dasari et al. [17] reported toughening of $\mathrm{PP}$ and $\mathrm{PP} / \mathrm{CaCO}_{3}$ nanocomposites by introducing submicrometer voids in the PP matrix without sacrificing its elastic properties. It was ascribed to the energy dissipated by debonding of the $\mathrm{CaCO}_{3}$ particles and, more significantly, the energies associated with plastic void growth in the PP matrix based on both pre-existent voids and those created by particle debonding, as well as void coalescence. Jiang et al. [14] compared toughening mechanisms in nano-sized precipitated calcium carbonate (NPCC) and organically modified montmorillonite (MMT) clay - PLA nanocomposites. Large quantities of microvoids were created in both PLA nanocomposites due to debonding at the polymer/nanoparticle interface. The microvoids in PLA/NPCC caused massive crazing, while in PLA/MMT they resulted in shear yielding as the MMT particles located between the microvoids prevented them from coalescing. Considering the role of adhesive strength between rigid filler particles and polymer, Thio et al. [18] showed that weaker adhesion yields earlier and more prevalent debonding that ultimately translates into higher macroscopic toughness. On the other hand, particles without any interfacial chemical bonds were not efficient in 
toughening [19]. It is interesting to note that high toughness was also observed for CNF paper [20] with porosity ranging from $19 \%$ to $40 \%$. The CNF paper toughness was dependent on the nanofibre network structure and nanofibre molar mass.

Reports on effect of foaming on the PLA strain at break and toughness are mixed. Some authors observed negligible [21] or significant negative effect [22-24] of foaming on the PLA strain at break and specific toughness (toughness divided by density), explained by the decrease in effective load bearing area and/or the large cells acting as stress concentrators [25]. On the other hand, Matuana [26] reported twofold increase in the PLA strain at break after foaming and up to 15.1 fold increase was achieved when ultrasonic irradiation during the nucleation stage of the foaming process was used $[27,28]$. The mechanism of the toughening was however not elucidated in the paper. Interestingly, in some cases foaming decreased the toughness in neat polymer while increased toughness was observed in its nanocomposite counterpart [25, 29]. In this case, toughness increase was attributed to finer cell morphology in the nanocomposite foam and weak fibre-matrix interface was concluded to be more efficient for toughening conversely to the stiffness enhancement [29].

The role of cell size in the toughening of cellular polymers is not clear. Miller and Kumar [30] showed that nanocellular PEI outperformed by far microcellular PEI in term of toughness. Increasing fracture toughness with increasing porosity was reported in thin nanoporous PAE films compared to their solid counterpart [31]. On the other hand, Weller and Kumar [32] did not detect a significant effect of the cell size ranging from $2.8 \mu \mathrm{m}$ to $37 \mu \mathrm{m}$ on tensile behaviour of microcellular polycarbonate. The tensile toughness of cellular nanocomposites therefore depends on many parameters, the foam morphology, the presence of submicron voids together with the interactions, either physical or chemical, between the nanoparticle and matrix and/or between nanoparticles themselves being the most important.

In this work, we studied the effect of CNF on the tensile stiffness, strength and toughness of microcellular PLA. Fully amorphous grade of PLA was selected as the presence of CNF [10] as well as the use supercritical $\mathrm{CO}_{2}[33,34]$ affect the crystallization kinetics of semi-crystalline PLA, the degree of crystallinity itself affecting the foam mechanical properties. Effects of the type of CNF (native or acetylated) and cellular morphology on the foam mechanical properties are discussed.

\section{Experimental}

\subsection{Materials}


Special grade of fully amorphous PLA resin which does not crystalize under the temperature Ingeo $4060 \mathrm{D}\left(T_{\mathrm{g}}=55-60^{\circ} \mathrm{C}, T_{\mathrm{m}}=210^{\circ} \mathrm{C}\right)$ was purchased from NatureWorks. Acetic acid, perchloric acid, and acetic anhydride were obtained from Kanto Chemicals Co., Inc., Tokyo, Japan, and toluene was obtained from Wako Pure Chemical Industries Ltd., Japan. Carbon dioxide, purity $>99.5 \%$, was supplied by Kyoto Teisan Co., Japan. All chemicals were used as received.

\subsection{Preparation of nanocomposites}

Native and acetylated CNF (ac-CNF, degree of substitution 0.44 ) were prepared from bleached never dried Kraft pulp by two passes fibrillation of $\sim 0.5 \%$ water suspension on a grinder (Masuko Sangyo Co.) at $1500 \mathrm{rpm}$. Nanocomposites containing $2.7 \mathrm{wt} \%$ and $9 \mathrm{wt} \%$ native or acetylated CNF (hereafter designated as 3CNF, 9CNF, 3ac-CNF, and 9ac-CNF) were prepared by a casting-kneading-hot pressing process using acetone as a solvent. Details of the acetylation procedure and nanocomposite preparation method are described in our previous paper [35].

\subsection{Foaming of nanocomposites}

Neat PLA and nanocomposite foams were prepared with a batch foaming technique using supercritical $\mathrm{CO}_{2}$ as a plasticiser and blowing agent. Strips cut from hot-pressed sheets $(35 \times 4$ $\times 0.5 \mathrm{~mm}$ ) were used for foaming. Each strip was placed in the opening of a rectangular mould (1 mm thickness) sandwiched between two metal plates as showed in Fig. 1. The plates were fixed using six screws. Because foaming ratio in nanocomposites is affected by the presence of CNF network, nanocomposites with higher CNF content exhibited lower foaming ratio as already observed in some previous studies [36]. As mechanical properties of foams scale with the foam density [5], two different neat PLA foams were prepared to serve as a comparison to 3CNF foams and 9CNF foams. Low density neat PLA denoted as Neat-1 was prepared using 1 mm thick mould while high density neat PLA (Neat-2) was prepared using $0.7 \mathrm{~mm}$ thick mould. Samples in the mould were placed in the pressure vessel and soaked in supercritical $\mathrm{CO}_{2}$ at $60^{\circ} \mathrm{C}$ for $6 \mathrm{~h}$ at a given pressure. Nucleation process was then initiated by a rapid pressure quench to atmospheric pressure. Foaming pressure ranged from 12 to $20 \mathrm{MPa}$. The aim was to achieve different cell morphology and investigate the effect of cell size on the foam properties. The foaming device (TSC-05-A2) was purchased from Taiatsu Techno Co., Tokyo, Japan. The maximum working temperature was $350^{\circ} \mathrm{C}$ and the limit pressure was $20 \mathrm{MPa}$. At least six samples were foamed for each nanocomposite; five for mechanical tests and one for morphology observation. 


\subsection{Field emission scanning electron microscope (FE-SEM) observations}

Foamed samples were freeze-fractured in liquid nitrogen and their cross-sections were examined on a JEOL JSM-6700F FE-SEM. All surfaces were coated with platinum to avoid charging under the electron beam. Five images were recorded to characterise each sample. For foaming pressures of $13 \mathrm{MPa}$ and $20 \mathrm{MPa}$, used for examination of tensile properties, two samples were analyzed under FE-SEM. For other foaming pressures only one sample was used to compare the morphology of foams obtained in the mould with the morphology of previously reported free foamed samples [35].

The cell density reported with respect to the initial solid polymer volume was determined as follows:

$$
N=\left(\frac{n}{A}\right)^{\frac{3}{2}} \cdot V_{f}
$$

where $N$ is the cell density, $n$ is the number of cells as counted on a FE-SEM micrograph, $A$ is the analysed area in $\mathrm{cm}^{2}$, and $V_{f}$ denotes the foaming ratio (ratio of the foamed and solid volume). The cell density and cell size were obtained using ImageJ software.

\subsection{TEM observation}

Staining was carried out in the vapor phase. The trimmed specimen embedded in epoxy resin was stained with solid $\mathrm{RuO}_{4}$ for 20 min in a sealed glass tube. The amorphous region and functional groups of the specimen became hard by staining and it was easily sectioned at room temperature. Leica Ultracut UCT type ultramicrotome equipped with a precision diamond knife $\left(45^{\circ}\right)$ Diatome was used for specimen sectioning. TEM observation was carried out using a JEOL JEM 1230 instrument at accelerated voltage of $120 \mathrm{kV}$.

\subsection{Measurement of bulk foam density and tensile testing}

The bulk foam density was measured using the buoyancy method with triethylene glycol as a reference liquid with known density. No obvious liquid uptake by the foamed samples was observed. Solid nanocomposites and foams prepared at $13 \mathrm{MPa}$ and 20MPa were also used to characterize tensile properties. Strips cut from hot-pressed sheets $(35 \times 4 \times 0.5 \mathrm{~mm})$ and their foamed counterparts $(\sim 35 \times 5 \times 1 \mathrm{~mm})$ were subjected to tensile tests conducted on a universal mechanical testing machine Instron 3365. The specimen gauge length was $20 \mathrm{~mm}$ and the cross-head speed was set to $5 \mathrm{~mm} \mathrm{~min}^{-1}$. Tensile toughness, which is the work of fracture per unit volume of the specimen, was obtained by integrating the area under the stress-strain curve. 
Three specimens were used to characterize the solid nanocomposites and five specimens to characterize the foamed materials. Both types of nanocomposites were stabilized in an airconditioned room $\left(23{ }^{\circ} \mathrm{C}, 65 \% \mathrm{RH}\right)$ for two weeks prior to testing to test the samples at same moisture content and avoid transitory effects of residual $\mathrm{CO}_{2}$ content on the foam properties [37]. To assess the effect of CNF and foaming condition on the tensile properties of foams, $t-$ test was performed and significance level was set to 0.05 .

\section{Results and discussion}

\subsection{Foam morphology}

\subsubsection{Effect of the mould on the foam morphology}

In the previous paper, we analysed the morphology of microcellular PLA nanocomposites obtained in free condition [35]. In this work, the same solid material was foamed in a simple mould; displayed in Fig. 1; to keep the rectangular shape and enable tensile testing. To assess the effect of the mould on foam morphology, we compared the cell size and cell density of freely foamed samples with samples prepared in the mould. Relative change in cell size and cell density due to the mould is shown in Fig. 2 where the cell size and cell density ratios are plotted per foam type and foaming condition. The use of mould clearly improved the cell morphology of the neat PLA and 3CNF composite foams while only a slight effect was observed for nanocomposite foams with a higher CNF loading. The cell size was reduced by $35.1 \%$ in the neat PLA foam, $32.1 \%$ in the 3 CNF composite foam and by $30.7 \%$ in the 3 ac-CNF composite foam for the moulded specimen as compared to free-formed samples. The cell size reduction was accompanied by a cell density increase. The cell density was in average 1.8 times higher in the neat PLA foam and 1.35 and 1.31 higher in the 3CNF and 3ac-CNF composite foams, respectively. Composite foams with $9 \%$ of CNF were less sensitive to the mould presence: while a slight decrease in cell size (12.6\% in the 9CNF and $10.6 \%$ in 9ac-CNF foam) was observed, the difference in cell density of free-foamed and moulded specimens was not statistically significant.

Positive effect of a mould use on the cell size was already observed by Arora et al. [38]. The mould use is not expected to affect the nucleation process as during the initial foaming stage the sample is not constrained by the mould. However, once the expanding sample becomes constrained by the mould plates, diffusion of $\mathrm{CO}_{2}$ from the sample surface is stopped which may affect the foam morphology [39]. We can also expect the effect of compressive stress exerted by the mould on the expanding polymer and quicker cooling of the sample due to the 
contact with metal plates. The fate (growth and coalescence) of nucleated bubbles depends on the remaining $\mathrm{CO}_{2}$ content not consumed during the nucleation stage, the rheological properties of softened polymer and the time available for bubble expansion before the polymer solidification. Higher amount of $\mathrm{CO}_{2}$ trapped in the polymer may lead to higher coalescence rate due to further softening of expanding polymer. Fig. 2 shows that in particular for the neat PLA and 3CNF foams, the cell density is higher when foams are prepared in the mould indicating that the cell coalescence was not promoted by the mould use.

\subsubsection{Effect of CNF on the foam morphology}

Following comments concern exclusively the foams prepared in the mould and are introduced to investigate the relationship between the foam cellular morphology and its mechanical properties. Fig. 3 shows the distribution of cell size for all foams prepared at 13MPa. Average values for both foaming conditions are summarized in Table 2. The nanocomposite foams exhibited smaller average cell size and reduced cell size distribution in comparison with neat PLA foams. It may be attributed to different nucleation and cell growth dynamics in function of the CNF type and loading as detailed in our previous paper [35]. Further, surface acetylation of CNF lead to better dispersion of ac-CNF in the PLA matrix as illustrated in Fig. 4 which also contributed to improved morpohology of ac-CNF composites. Reduction in average cell size and narrowed cell size distribution observed in the PLA-CNF foams are in agreement with the previous studies on the nanoparticles' effect on the foam morphology as for example the nanoclay effect reported by Ema et al. [40].

\subsection{Mechanical properties of solid and foamed PLA and its nanocomposites}

\subsubsection{Tensile properties of solid nanocomposites}

Table 1 summarizes the tensile properties of solid PLA and PLA-CNF nanocomposites. Elastic modulus of solid PLA was significantly enhanced when $9 \%$ of native or acetylated CNF were introduced in the PLA matrix but the difference in stiffness due to the CNF type used was not significant for the stiffness enhancement. Conversely, 9ac-CNF nanocomposite exhibited significantly higher strength when compared to 9CNF nanocomposite. Higher strengthening effect of ac-CNF may be explained by better dispersion of ac-CNF in the PLA matrix (Fig. 4) due to higher fibre-matrix affinity. As reported by Tingaut et al. [11], achieving of optimal degree of CNF acetylation results in improved compatibility at the cellulose filler-PLA matrix interface and so improved dispersion. Introduction of CNF in the PLA matrix further lead to increase in brittleness. The strain at break dropped from $8.6 \pm 0.7 \%$ in the neat PLA to around 
$3 \%$ in their nanocomposite counterparts. These results are consistent with previous reports on the PLA-CNF nanocomposites prepared by the casting/kneading/hotpressing process or by extrusion [8-10].

\subsubsection{Density and tensile properties of foamed nanocomposites}

Density and tensile properties of PLA-CNF foams are summarised in Table 2. Bulk foam density ranged between $0.33 \mathrm{~g} \mathrm{~cm}^{-3}$ and $0.64 \mathrm{~g} \mathrm{~cm}^{-3}$ and increased with the amount of CNF. To assess the effect of CNF regardless of the density differences between foams, the specific values of the tensile properties i.e. divided by density were considered in the following. Similarly to solid nanocomposites (Table 1), introduction of CNF resulted in the foam stiffening and strengthening however the magnitude of CNF effect was significantly enhanced by foaming. For example, the addition of $9 \%$ of ac-CNF increased the specific modulus and specific strength by $19 \%$ and $20 \%$ respectively in solid nanocomposites while $44 \%$ respectively $46 \%$ increase was observed in their foamed counterparts (foams prepared at $20 \mathrm{MPa}$, taking Neat-2 as a reference). This likely resulted from the combined effect of CNF on the cell wall properties and the foam morphology. The specific modulus of foamed nanocomposites significantly increased with the CNF content and for the 9CNF composite also with surface acetylation. Specific strength was enhanced in nanocomposite foams when compared to neat PLA foams but differences due to the CNF content or surface modification were not statistically significant. Considering the effect of foaming pressure on the foam properties, all tensile properties increased with increase in foaming pressure for Neat-1 PLA. Conversely, the tensile properties of nanocomposite foams were not affected by the foaming condition except for the strain at break in the 9CNF nanocomposites. This difference in tensile properties is likely related to the decrease in open cell content in the foams prepared at 20MPa (cf Table 2). The effect of open cell content on the foam properties will be discussed in more details in the

\subsection{3 section.}

The most notable change in tensile properties after foaming was the increase in strain at break and so in tensile toughness. Strain at break increased up to 7.5 times in the neat PLA and up to 31.5 times in the $3 \mathrm{CNF}$ composite foam. It is also interesting to note the difference between the high deformability of 9CNF foam (maximum strain at break is 55\%) contrasting with more brittle fracture of 9ac-CNF composite foam (maximum strain at break is $10.9 \%$ ).

\subsubsection{Parameters affecting the tensile properties and comparison with literature data}


Mechanical properties of foams scale with relative density however other structural parameters such as the proportion of open and closed cells, cell size and its uniformity or plastic deformation of pre-existing and/or created microvoids may also affect the foam tensile behaviour. According to Gibson and Ashby [5], the strength of foams scale with relative density and proportion of open and closed cells as follows:

$$
\frac{\sigma_{f}}{\sigma_{c w}}=0.3 \cdot \phi^{\frac{3}{2}} \cdot\left(\frac{\rho_{f}}{\rho_{c w}}\right)^{\frac{3}{2}}+(1-\phi) \cdot\left(\frac{\rho_{f}}{\rho_{c w}}\right)
$$

where $\sigma$ is the yield strength, $\rho$ the density, and subscripts $f$ and $c w$ correspond to the foam and cell wall material, respectively. Parameter $\phi$ is the fraction of solid in the foam contained in the cell edges, and non-linear member of the above equation represents the contribution of cell edges to the strength. The second member with the $(1-\phi)$ parameter describes the contribution of the cell face.

Fig. 5a) shows the relationship between the relative foam strength and relative foam density. In addition to our result, data from two other papers are plotted: data by Ji et al. [28] on the tensile properties of microcellular foams blown by supercritical $\mathrm{CO}_{2}$ using ultrasonic irradiation to control the foaming process of different grades of semi-crystalline PLA and data gathered by Bergeret and Benezet [41] and re-analysed in Julien, Bénézet et al. [21] on the tensile properties of neat PLA and PLA-cellulose microfiber composite foams blown with different chemical agents. Further, the model trend lines predicting the behaviour of foams with various fractions of open cells are plotted. Considering foams prepared in the present study, nanocomposite foams exhibit lower fraction of open cells compared to the neat PLA foams. This indicates beneficial effect of CNF on the final foam structure likely resulting from the fact that CNF-PLA interface acts as a heterogeneous nucleating site leading to quicker consumption of the foaming gas [35]. Comparison with results from other studies suggests the importance of the foam processing way for the final foam properties. Neat PLA foams prepared by Ji et al. [28] follow the linear trend predicted for closed cell foams while foams prepared by Bergeret and Benezet [41] show significant deviation from the linear model indicating the presence of a large fraction of open cells. Bergeret and Benezet [41] observed higher open cell content in the nanocomposite foams when compared to neat PLA foams, conversely to results obtained in our study. Considering high amount of cellulose filler used (20 to 30\%), it is likely that agglomeration of fillers occurred which is confirmed by lower specific modulus obtained in 
nanocomposites when compared to neat PLA. Further, nanocomposite foams were prepared with different blowing agent than neat PLA foam giving similar foam morphology but significantly weaker mechanical properties [41], indicating again the effect of the foam processing on the open cell content.

Fig. 5b) shows the relationship between the strain at break and relative density. The relationship is not monotonous however when each type of foam is considered separately, strain at break exhibits strong linear relationship with relative density. There seems to be an optimal relative density suitable for achieving high flexibility of PLA foams but further investigations are necessary to confirm or infirm this statement. When compared to results obtained by Ji et al. [28], at a comparable relative density the flexibility of foams prepared in the present study is similar (neat PLA) or higher (nanocomposite foam) despite the fraction of open cells present in our material. It is interesting to note that nanocomposite foams follow different trend line when compared to neat PLA foam and higher strain at break is achieved in nanocomposite foams at similar foam density. This may be related to the difference in the foam morphology (open cell content, cell size and cell size heterogeneity) and/or properties of the cell wall material itself. The effect of the open cell content on the flexibility and toughness of foams is shown in Fig. 5c) and d). High fraction of open cells in the foam clearly reduces the foam flexibility regardless the nature (nanocomposite or neat) of the foam. Similar observation may be done for the overall work of fracture which is closely related to the strain at break (the relationship with open cell content is stronger than with relative density for which the $\mathrm{R}^{2}$ is of 0.31 ; further open cell content is not related to relative density giving $\mathrm{R}^{2}$ of 0.1 ). If the relative density is the first order parameter affecting the foam properties, open cell content is an important structural parameter to take into account not only for the foam strength but also for the foam flexibility. Larger cell size and cell size heterogeneity were reported to be detrimental to the PLA foam mechanical performances, in particular to the strain at break [27]. In our study, no significant relationship between the strain at break or work of fracture and cell size was observed (not shown, re-analysis of data by Ji et al. [28] lead to the same conclusion). However, the wider cell size distribution observed in the neat PLA when compared to nanocomposite foams (Fig. 3) may contribute, together with the higher open cell content, to lower flexibility and overall toughness observed in neat PLA foams.

Fig. 5d) and table 2 shows that the strain at break and toughness of 9ac-CNF foams is significantly lower when compared to 9CNF foams. The obvious brittleness of 9ac-CNF foams 
can not be explained by difference in the cell morphology such as the open cell content (similar or higher in 9CNF foams) or cell size (relationship with strain at break not significant) but is likely related to the inherent properties of cell wall material, in particular the interfacial strength between the CNF and PLA matrix. Some previous studies reported significant increase in the polymer strain at break and consequently tensile toughness after foaming $[7,26]$ and/or with addition of nanoparticles [12,13,17-19]. This toughening effect was attributed to the energy dissipated during the debonding of the nanoparticle from the polymer matrix and, more significantly, the energies associated with the plastic growth of submicron voids pre-existing in the polymer matrix or created from the nanoparticle debonding during tensile testing. We can see from Fig. 4 that 3ac-CNF foam contains some submicron voids while in 3CNF foam, only cells of several micrometers are present. It is hypothesized that the absence of pre-existing microvoids in the PLA-CNF foams may be compensated by lower interfacial strength between the PLA matrix and native CNF which allows for earlier yielding and more prevalent debonding at the PLA-CNF interface according to observation by Thio et al. [18] who reported that weaker rigid filler - matrix interface is more efficient in energy dissipation. This may explain the higher work of fracture observed for 9CNF composite foam compared with their acCNF counterparts. Further study including the SEM and TEM analysis of the fracture surface is however needed to elucidate the deformation mechanism of microcellular PLA-CNF foams as well as the relative contribution of plastic deformation of pre-existing and created submicron voids. In conclusion, relative foam density, the fraction of open cells and strength of the PLACNF interface seem to be key parameters affecting the strength but also the flexibility of nanocomposite foams. In future, it would be interesting to submit nanocomposite foams to compressive as well as cyclic tests to get further insights into the interaction between the CNF network and the PLA foamed matrix under different type of loading.

\section{Conclusions}

In this paper, we investigated the tensile properties of solid and microcellular PLA reinforced with native and acetylated CNF. It was shown that the effect of CNF on the specific modulus, specific strength and strain at break was considerably enhanced by foaming when compared to their effect in their solid counterparts. The higher CNF content and the use of ac-CNF further promoted the positive CNF effect on the specific modulus of nanocomposite foams while specific strength was not significantly affected by the content and the type of CNF. Major issue 
of the paper is the large increase in strain at break and so overall tensile toughness of the PLA and its nanocomposites after foaming. Up to 7.5 fold and 31.5 fold increase in the strain at break was observed for the neat PLA and 3CNF nanocomposite respectively. In addition to relative density, fraction of open cells in the foam revealed to be an important structural parameter affecting the foam strength and flexibility while differences in cell size did not significantly affect the foam tensile properties. While the use of $9 \%$ of ac-CNF imparted high elastic modulus and strength to the PLA foams, 9CNF-PLA foams exhibited high flexibility and toughness indicating that the strength of CNF-PLA interface may play an important role in the deformational behaviour of nanocomposite foams.

\section{Acknowledgments}

The first author sincerely thanks the Japanese Society of Promotion of Science for supporting this work. Authors would also like to acknowledge Dr. Hironari Sano from Mitsubishi Chemical Corporation, Japan for his precious help with TEM observations. The UMR 1092 LERFoB is supported by the French National Research Agency through the Laboratory of Excellence ARBRE (ANR-12- LABXARBRE-01).

\section{References}

[1] R.M. Rasal, A.V. Janorkar, D.E. Hirt, Progress in Polymer Science, 35 (2010) 338-356.

[2] D.I. Collias, D.G. Baird, R.J.M. Borggreve, Polymer, 35 (1994) 3978-3983.

[3] C. Barlow, V. Kumar, B. Flinn, R.K. Bordia, J. Weller, Journal of Engineering Materials and Technology-Transactions of the Asme, 123 (2001) 229-233.

[4] S. Doroudiani, C.B. Park, M.T. Kortschot, Polymer Engineering \& Science, 38 (1998) 1205-1215.

[5] L.J. Gibson, M.F. Ashby, Cellular solids. Structure and properties, 2ème ed. (1ère ed. 1988) ed., Cambridge University Press, Cambridge, 1997.

[6] H.B. Zhang, Q. Yan, W.G. Zheng, Z.X. He, Z.Z. Yu, Acs Applied Materials \& Interfaces, 3 (2011) 918-924.

[7] C. Zeng, N. Hossieny, C. Zhang, B. Wang, S.M. Walsh, Composites Science and Technology, 82 (2013) 29-37.

[8] A. Iwatake, M. Nogi, H. Yano, Composites Science and Technology, 68 (2008) 2103-2106. 
[9] M. Jonoobi, J. Harun, A.P. Mathew, K. Oksman, Composites Science and Technology, 70 (2010) 1742-1747.

[10] L. Suryanegara, A.N. Nakagaito, H. Yano, Composites Science and Technology, 69 (2009) 1187-1192.

[11] P. Tingaut, T. Zimmermann, F. Lopez-Suevos, Biomacromolecules, 11 (2010) 454-464.

[12] M. Bulota, K. Kreitsmann, M. Hughes, J. Paltakari, Journal of Applied Polymer Science, 126 (2012) E449-E458.

[13] M. Bulota, M. Hughes, Journal of Materials Science, 47 (2012) 5517-5523.

[14] L. Jiang, J. Zhang, M.P. Wolcott, Polymer, 48 (2007) 7632-7644.

[15] R. Bagheri, R.A. Pearson, Polymer, 37 (1996) 4529-4538.

[16] P. Ma, D.G. Hristova-Bogaerds, J.G.P. Goossens, A.B. Spoelstra, Y. Zhang, P.J. Lemstra, European Polymer Journal, 48 (2012) 146-154.

[17] A. Dasari, Q.-X. Zhang, Z.-Z. Yu, Y.-W. Mai, Macromolecules, 43 (2010) 5734-5739.

[18] Y.S. Thio, A.S. Argon, R.E. Cohen, Polymer, 45 (2004) 3139-3147.

[19] N.C. Liu, W.E. Baker, Polymer, 35 (1994) 988-994.

[20] M. Henriksson, L.A. Berglund, P. Isaksson, T. Lindstro $\square$ m, T. Nishino,

Biomacromolecules, 9 (2008) 1579-1585.

[21] J.M. Julien, J.C. Bénézet, E. Lafranche, J.C. Quantin, A. Bergeret, M.F. Lacrampe, P. Krawczak, Polymer, 53 (2012) 5885-5895.

[22] S. Pilla, A. Kramschuster, J. Lee, C. Clemons, S.Q. Gong, L.S. Turng, Journal of Materials Science, 45 (2010) 2732-2746.

[23] S. Pilla, A. Kramschuster, L. Yang, J. Lee, S. Gong, L.-S. Turng, Materials Science and Engineering: C, 29 (2009) 1258-1265.

[24] A. Ameli, D. Jahani, M. Nofar, P.U. Jung, C.B. Park, Journal of Cellular Plastics, (2013).

[25] A. Kramschuster, S. Gong, L.S. Turng, T. Li, T. Lil, Journal of Biobased Materials and Bioenergy, 1 (2007) 37-45.

[26] L.M. Matuana, Bioresource Technology, 99 (2008) 3643-3650.

[27] J. Wang, W. Zhai, J. Ling, B. Shen, W. Zheng, C.B. Park, Industrial \& Engineering Chemistry Research, 50 (2011) 13840-13847.

[28] G. Ji, J. Wang, W. Zhai, D. Lin, W. Zheng, Journal of Cellular Plastics, 49 (2013) 101117. 
[29] A. Javadi, Y. Srithep, J. Lee, S. Pilla, C. Clemons, S.Q. Gong, L.S. Turng, Composites Part a-Applied Science and Manufacturing, 41 (2010) 982-990.

[30] D. Miller, V. Kumar, Polymer, 52 (2011) 2910-2919.

[31] A.V. Kearney, C.S. Litteken, C.E. Mohler, M.E. Mills, R.H. Dauskardt, Acta Materialia, 56 (2008) 5946-5953.

[32] J.E. Weller, V. Kumar, Polymer Engineering \& Science, 50 (2010) 2170-2175.

[33] M. Mihai, M.A. Huneault, B.D. Favis, Journal of applied polymer science, 113 (2009) 2920-2932.

[34] D.-c. Li, T. Liu, L. Zhao, X.-s. Lian, W.-k. Yuan, Industrial \& Engineering Chemistry Research, 50 (2011) 1997-2007.

[35] J. Dlouha, L. Suryanegara, H. Yano, Soft Matter, 8 (2012) 8704-8713.

[36] C.I.R. Boissard, P.-E. Bourban, P. Tingaut, T. Zimmermann, J.-A.E. Månson, Journal of Reinforced Plastics and Composites, 30 (2011) 709-719.

[37] D.I. Collias, D.G. Baird, Polymer Engineering and Science, 35 (1995) 1167-1177.

[38] K.A. Arora, A.J. Lesser, T.J. McCarthy, Macromolecules, 31 (1998) 4614-4620.

[39] S. Siripurapu, J.A. Coughlan, R.J. Spontak, S.A. Khan, Macromolecules, 37 (2004) 98729879.

[40] Y. Ema, M. Ikeya, M. Okamoto, Polymer, 47 (2006) 5350-5359.

[41] A. Bergeret, J.C. Benezet, International Journal of Polymer Science, 2011 (2011) 14.

\section{Figure captions:}

Fig. 1. Mould used for foaming: PLA or nanocomposite strips were placed in the mould opening and sandwiched between the two metal plates using six screws.

Fig. 2. Ratio of cell size and cell density of foams obtained in the mould and in free condition prepared at different foaming pressures. The neat PLA considered in this section corresponds to Neat-1 in the section concerned with mechanical properties.

Fig. 3. Cell size distribution in the neat PLA and its nanocomposite foams prepared at 13MPa.

Fig. 4. TEM micrographs showing the morphology and dispersion of CNF in $3 \mathrm{CNF}(a, b)$ and $3 \mathrm{ac}-\mathrm{CNF}(\mathrm{c}, \mathrm{d})$ nanocomposite foams prepared at a foaming pressure of $20 \mathrm{MPa}$.

Fig. 5. Relationship between a) relative strength and relative density; b) strain at break and relative density; c) strain at break and open cell content and d) work of fracture and open cell 
content. Trend lines in Fig. a) correspond to the relative strength of foam with a given open cell content $(\phi)$ as predicted by Eq. 2 Neat and Nc denotes neat PLA and nanocomposite foam, respectively. Red points correspond to measurements of the present study, black and blue points to bibliographic data: $* 1$ is extracted from [41] and *2 from [28].

\section{Tables}

Table 1 Mechanical properties of solid nanocomposites. $\rho$ is density, $E$ is elastic modulus, $\sigma$ is tensile strength, $\varepsilon$ is strain at break, and $W_{A}$ is tensile work of fracture.

\begin{tabular}{lccccccc} 
Solid & $\rho_{f}\left(\mathrm{~g} \mathrm{~cm}^{-3}\right)$ & $E(\mathrm{MPa})$ & $\sigma(\mathrm{MPa})$ & $E / \rho_{\mathrm{f}}(\mathrm{MPa})$ & $\sigma / \rho_{\mathrm{f}}(\mathrm{MPa})$ & $\varepsilon(\%)$ & $\mathrm{W}_{\mathrm{A}}\left(\mathrm{MJ} \mathrm{m} \mathrm{m}^{-3}\right)$ \\
\hline Neat & $1.26 \pm 0.01$ & $2455 \pm 34$ & $51.3 \pm 0.1$ & $1949 \pm 27$ & $40.7 \pm 0.1$ & $8.6 \pm 0.7$ & $3.6 \pm 0.3$ \\
3CNF & $1.27 \pm 0.01$ & $2646 \pm 157$ & $54.6 \pm 0.8$ & $2083 \pm 124$ & $43.0 \pm 0.6$ & $3.2 \pm 0.5$ & $1.1 \pm 0.2$ \\
3ac-CNF & $1.26 \pm 0.01$ & $2645 \pm 34$ & $54.6 \pm 0.7$ & $2099 \pm 27$ & $43.3 \pm 0.6$ & $3.1 \pm 0.1$ & $1.0 \pm 0.0$ \\
9CNF & $1.28 \pm 0.01$ & $2876 \pm 207$ & $57.1 \pm 0.7$ & $2247 \pm 162$ & $44.6 \pm 0.6$ & $2.6 \pm 0.3$ & $0.9 \pm 0.1$ \\
9ac-CNF & $1.27 \pm 0.01$ & $3068 \pm 95$ & $61.5 \pm 1.7$ & $2416 \pm 75$ & $48.4 \pm 1.3$ & $2.9 \pm 0.4$ & $0.9 \pm 0.1$ \\
\hline
\end{tabular}

Table 2 Mechanical properties of foamed nanocomposites. $P$ is foaming pressure, $\rho_{f}$ is foam density, $\phi$ is the open cell content and for other symbols the legend is the same as for Table 1 .

\begin{tabular}{|c|c|c|c|c|c|c|c|c|c|}
\hline $\mathrm{P}=13 \mathrm{MPa}$ & $\rho_{\mathrm{f}}\left(\mathrm{g} \mathrm{cm}^{-3}\right)$ & $\mathrm{E}(\mathrm{MPa})$ & $\sigma(\mathrm{MPa})$ & $E / \rho_{f}(\mathrm{MPa})$ & $\sigma / \rho_{f}(\mathrm{MPa})$ & $\varepsilon(\%)$ & $\mathrm{W}_{\mathrm{A}}\left(\mathrm{MJ} \mathrm{m}^{-3}\right)$ & $\begin{array}{l}\text { Cell size } \\
(\mu \mathrm{m})\end{array}$ & $\phi$ \\
\hline Neat-1 & $0.33 \pm 0.02$ & $327 \pm 27$ & $5.6 \pm 0.3$ & $1001 \pm 82$ & $17.2 \pm 1.1$ & $35.5 \pm 12.9$ & $1.8 \pm 0.7$ & $31.4 \pm 8.4$ & 0.68 \\
\hline Neat-2 & $0.64 \pm 0.07$ & $992 \pm 108$ & $12.9 \pm 1.2$ & $1540 \pm 70$ & $20.0 \pm 1.3$ & $13.2 \pm 4.9$ & $1.5 \pm 0.5$ & $27.1 \pm 6.7$ & 0.65 \\
\hline $3 \mathrm{CNF}$ & $0.42 \pm 0.01$ & $675 \pm 24$ & $10.8 \pm 0.2$ & $1610 \pm 33$ & $25.8 \pm 0.2$ & $77.4 \pm 4.3$ & $7.8 \pm 0.5$ & $9.5 \pm 2.3$ & 0.48 \\
\hline 3ac-CNF & $0.45 \pm 0.02$ & $865 \pm 65$ & $12.4 \pm 0.5$ & $1923 \pm 151$ & $27.7 \pm 1.7$ & $95.0 \pm 19.0$ & $10.5 \pm 1.8$ & $6.2 \pm 1.6$ & 0.44 \\
\hline 9CNF & $0.55 \pm 0.03$ & $999 \pm 39$ & $13.7 \pm 0.2$ & $1832 \pm 53$ & $25.1 \pm 1.6$ & $31.7 \pm 2.3$ & $4.1 \pm 0.3$ & $6.6 \pm 1.7$ & 0.57 \\
\hline 9ac-CNF & $0.61 \pm 0.03$ & $1290 \pm 88$ & $17.6 \pm 1.8$ & $2118 \pm 66$ & $28.9 \pm 2.1$ & $10.9 \pm 6.0$ & $1.8 \pm 1.2$ & $4.3 \pm 1.5$ & 0.51 \\
\hline $\mathrm{P}=20 \mathrm{MPa}$ & $\rho_{\mathrm{f}}\left(\mathrm{g} \mathrm{cm}^{-3}\right)$ & $\mathrm{E}(\mathrm{MPa})$ & $\sigma(\mathrm{MPa})$ & $\mathrm{E} / \mathrm{\rho}_{\mathrm{f}}(\mathrm{MPa})$ & $\sigma / \rho_{\mathrm{f}}(\mathrm{MPa})$ & $\varepsilon(\%)$ & $\mathrm{W}_{\mathrm{A}}\left(\mathrm{MJ} \mathrm{m}^{-3}\right)$ & $\begin{array}{c}\text { Cell size } \\
(\mu \mathrm{m})\end{array}$ & $\phi$ \\
\hline Neat-1 & $0.34 \pm 0.02$ & $486 \pm 24$ & $7.2 \pm 0.4$ & $1412 \pm 39$ & $21.0 \pm 1.2$ & $64.3 \pm 15.2$ & $4.3 \pm 1.1$ & $12.6 \pm 4.0$ & 0.57 \\
\hline Neat-2 & $0.61 \pm 0.05$ & $926 \pm 101$ & $11.9 \pm 1.2$ & $1529 \pm 173$ & $19.7 \pm 2.4$ & $17.4 \pm 5.7$ & $1.9 \pm 0.6$ & $13.4 \pm 3.6$ & 0.65 \\
\hline $3 \mathrm{CNF}$ & $0.38 \pm 0.02$ & $648 \pm 48$ & $10.3 \pm 0.6$ & $1729 \pm 106$ & $27.5 \pm 1.8$ & $100.6 \pm 14.5$ & $9.5 \pm 1.9$ & $6.3 \pm 1.8$ & 0.43 \\
\hline 3ac-CNF & $0.40 \pm 0.02$ & $737 \pm 45$ & $10.6 \pm 0.3$ & $1837 \pm 132$ & $26.5 \pm 1.4$ & $92.0 \pm 10.0$ & $8.9 \pm 1.3$ & $4.9 \pm 1.4$ & 0.47 \\
\hline 9CNF & $0.49 \pm 0.01$ & $984 \pm 54$ & $13.3 \pm 0.4$ & $1990 \pm 137$ & $26.9 \pm 1.3$ & $55.0 \pm 7.0$ & $6.9 \pm 1.1$ & $4.5 \pm 1.5$ & 0.49 \\
\hline 9ac-CNF & $0.60 \pm 0.02$ & $1328 \pm 57$ & $17.3 \pm 0.5$ & $2203 \pm 127$ & $28.6 \pm 1.4$ & $10.3 \pm 4.2$ & $1.6 \pm 0.7$ & $3.2 \pm 1.2$ & 0.51 \\
\hline
\end{tabular}




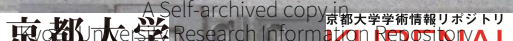

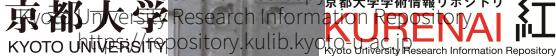

Upper plate

\section{Sample}

\section{Mould}



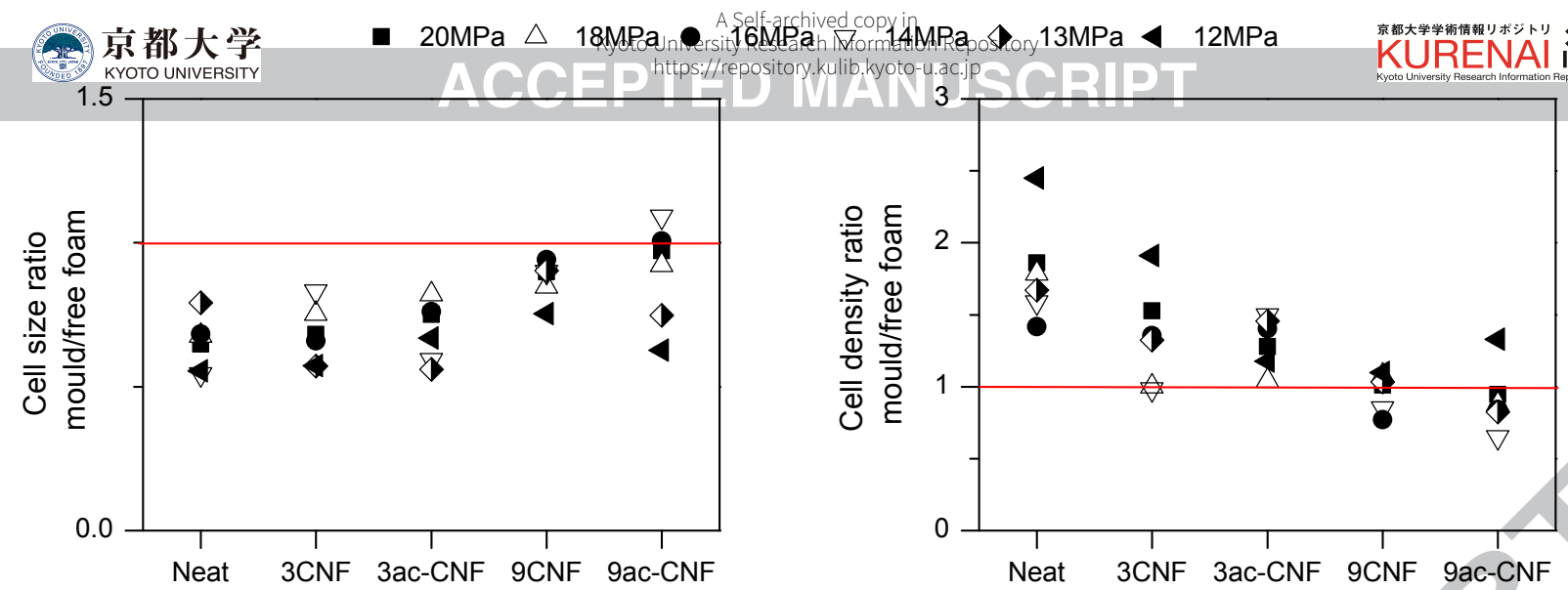
A Self-archived copy in 


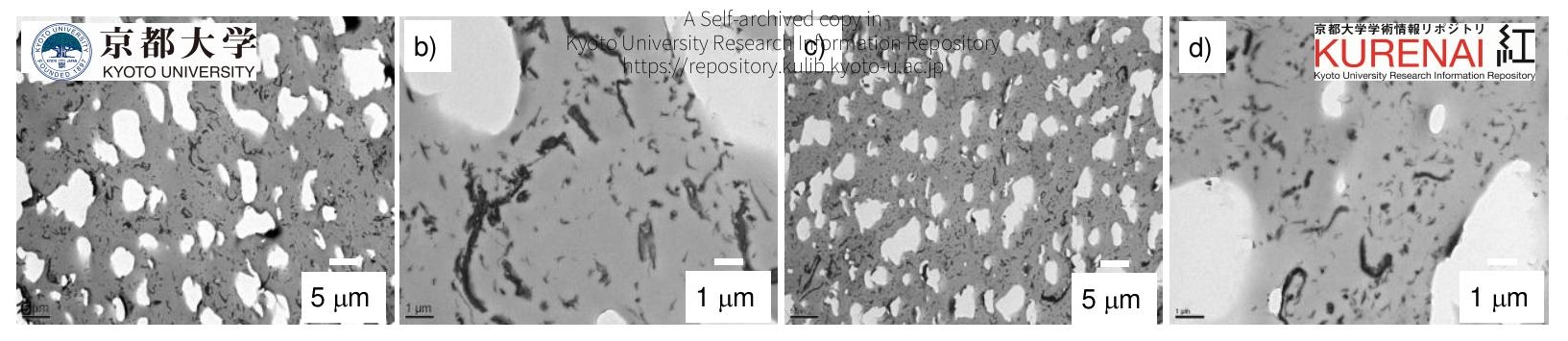




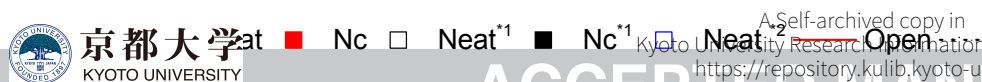

a)

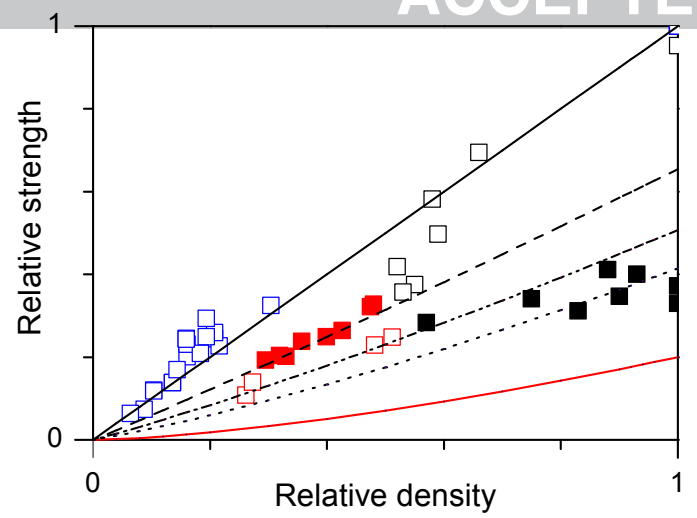

c)

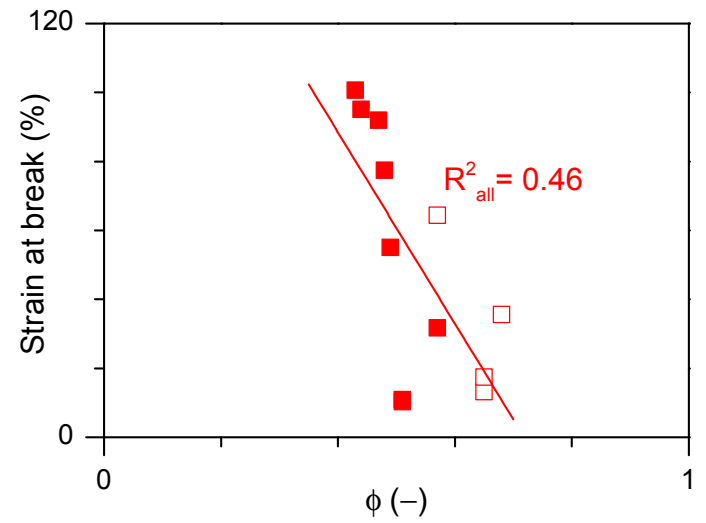

b)

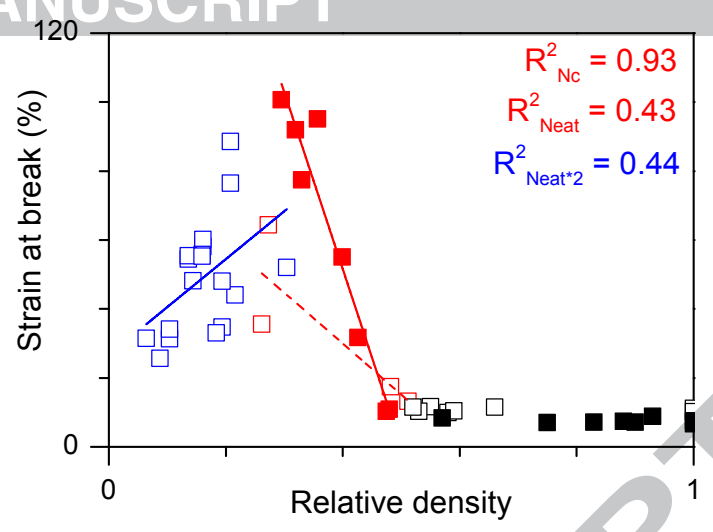

d)

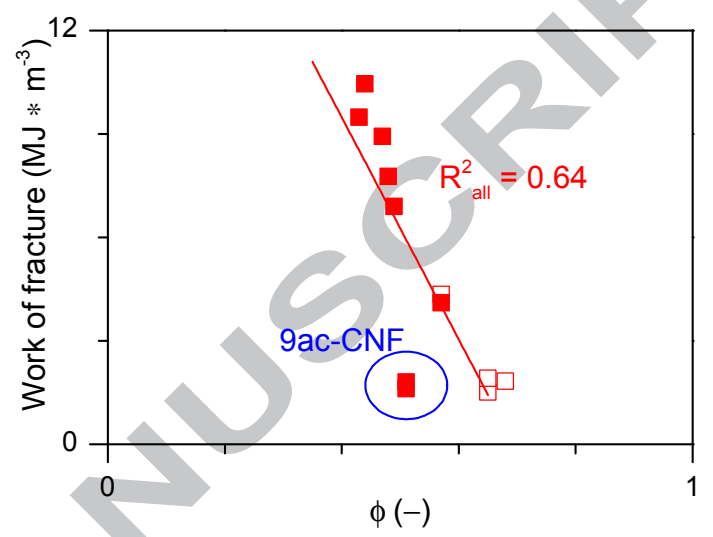

